\section{Physiotherapie bei systemischer Sklerose - Verschreibungsverhalten untersucht}

Belz D et al. Large Variability of Frequency and

Type of Physical Therapy in Patients in the

German Network for Systemic Sclerosis. Arthritis

Care \&. Research 2020; 72: 1041-1048

Welcher Art und Häufigkeit ist die, von Ärzten bei systemischer Sklerose verschriebene Physiotherapie. Welche Merkmale kennzeichnet die systemische Sklerose bei Patientinnen und Patienten, die eine Physiotherapie erhalten und wie sehen die Krankheitsmerkmale bei denjenigen aus, die keine erhalten?

Für die Studie werteten die Autorinnen und Autoren die Daten von mehr als $4500 \mathrm{~Pa}$ tientinnen und Patienten des Registers des Deutschen Netzwerks für Systemische Sklerose aus. Das Netzwerk wurde 2003 gegründet. An dem Netzwerk sind mehr als 40 rheumatologische und dermatologische Zentren beteiligt.

Das Durchschnittsalter der Patientengruppe zu Studienbeginn betrug 57,6 \pm 14,1 Jahren und zum Zeitpunkt des Auftretens der Er-

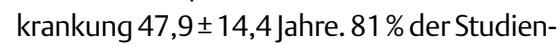
teilnehmer waren weiblich, $19 \%$ männlich. Insgesamt erhielten 37,4\% der Patientinnen und Patienten (1590 von 4252) eine Physiotherapie bis zum Ende einer jährlichen Nachuntersuchung verschrieben.

Der am häufigsten zur Anwendung kommende Typ der Physiotherapie war die Lymphdrainage bei 1061 (36,8\%) Personen, gefolgt von einer Bewegungstherapie in 1047 der Fälle (36,3\%) und der Wärmetherapie in 689 Fällen (23,9\%). Mehr als drei von 4 der behandelten Patientinnen und Patienten (82\%) erhielten gleichzeitig eine oder 2 verschiedene Formen einer Physiotherapie, $5 \%$ erhielten $\geq 4$ Formen einer Physiotherapie.

Eine detailliertere Analyse ergab, dass eine schwere Hautfibrose mit der Verschreibung einer Physiotherapie assoziiert war. Dabei wurden signifikante Unterschiede anhand des modifizierten Rodnan-Hautdicken- 
Scores (MRSS) gefunden. (MRSS < 10; $n=$ 910 von 2179 [41,8\%]; moderater MRSS 11-20; 401 von 718 [55,8\%], MRSS mit hoher Hautfibrose $>21$; 209 von 327 [63,9\%]).

Die Wahrscheinlichkeit der Verschreibung einer Physiotherapie war bei muskoloskelettaler Beteiligung (z. B. Arthritis, Muskelschwäche, Gelenkkontrakturen, Reibung der Sehnen) höher, als ohne diese Symptome. Die entsprechenden ORs lagen zwischen 1,96 (95\% Konfidenzintervall [KI] 1,69-2,28) für Gelenkkontrakturen und 3,83 (95\% KI 2,89-5,08) für Arthritis. Rheumatologen verschrieben eine Physiotherapie signifikant seltener als Dermatologen (29 vs. 56,8\%; $p<0,001$ ).

Beim Vergleich der Verschreibungen einer Physiotherapie beim ersten Besuch und allen Nachuntersuchungen von 2003 bis 2017 wurde festgestellt, dass die Zahl der Verschreibungen über diesen Zeitraum signifikant variierte, mit der höchsten Verschreibungsrate zwischen 2003 und 2005 (46\%) und der geringsten zwischen 2009 und 2011 (40,3\%). Eine leichte Erholung wurde zwischen 2015 und 2017 festgestellt (44,3\%).

\section{FAZIT}

Dies ist die erste Studie, die die Verschreibungen eine Physiotherapie bei Patientinnen und Patienten mit systemischer Sklerose systematisch erfasst, so das Autorenteam. Obwohl die Erkrankung durch erhebliche Behinderung und Bewegungseinschränkung gekennzeichnet ist, erhalten weniger als $40 \%$ dieser Patientinnen und Patienten eine Physiotherapie.

Richard Kessing, Zeiskam 